\title{
GLOBAL OPTIMIZATION METHOD FOR SOLVING THE MINIMUM MAXIMAL FLOW PROBLEM
}

\author{
JUN-YA GOTOH, NGUYEN VAN THOAI, AND YOSHITSUGU YAMAMOTO
}

\begin{abstract}
The problem of minimizing the flow value attained by maximal flows plays an important and interesting role to investigate how inefficiently a network can be utilized. It is a typical multiextremal optimization problem, which can have local optima different from global optima. We formulate this problem as an global optimization problem with a special structure and propose a method to combine different techniques in local search and global optimization. Within the proposed algorithm, tha advantageous structure of network flow is fully exploited so that the algorithm should be suitable for handling the problem of moderate sizes.
\end{abstract}

\section{INTRODUCTION}

Consider a directed network $N(V, E, s, t, c)$, where $V$ is the set of $m+2$ nodes, $E$ is the set of $n$ arcs, $s$ is the single source node, $t$ is the single sink node, and $c$ is the vector of arc capacities. A vector $x$ of dimension $n$ is said to be a feasible flow if it satisfies the system of conservation equations and capacity constraints:

$$
A x=0 ; 0 \leq x \leq c,
$$

where $A$ is the well-known node-arc incidence matrix restricted to the node set $V \backslash\{s, t\}$, whose size is then $m \times n$. We denote by $X$ the set of feasible flows, i.e.,

$$
X=\left\{x \mid x \in \mathbb{R}^{n} ; A x=0 ; 0 \leq x \leq c\right\} .
$$

A vector $x \in X$ is called a maximal flow if there does not exist $x^{\prime} \in X$ such that $x^{\prime} \geq x$ and $x^{\prime} \neq x$. We denote the set of all maximal flows by $X_{M}$. Further, let $\Delta^{+}(s)$ and $\Delta^{-}(s)$ denote the sets of arcs leaving and entering the source node $s$, respectively. Then the total amount of flow, called the flow value, of $x$ is given by

$$
\sum_{h \in \Delta^{+}(s)} x_{h}-\sum_{h \in \Delta^{-}(s)} x_{h}
$$

The problem to be considered in this article is the minimization of the flow value over the set of all maximal flows, i.e.,

Date: May 28, 2001.

Key words and phrases. minimum maximal flow, global optimization, optimization over efficient sets, adjacent vertex search, branch and bound.

This paper is written during a stay of the second author as a foreign professor at the Institute of Policy and Planning Sciences, University of Tsukuba, Japan.

The authors thank Dolf Talman, Tilburg University and Maiko Shigeno, University of Tsukuba for stimulating discussion.

The third author is supported by the Grant-in-Aid for Scientific Research C(2) 13650061 of the Ministry of Education, Culture, Sports, Science and Technology of Japan. 


$$
\begin{array}{l|ll}
P & \min & d x \\
\text { s.t. } & x \in X_{M},
\end{array}
$$

where $d$ is an $n$-dimensional row vector defined as

$$
d_{h}= \begin{cases}1 & \text { if } h \in \Delta^{+}(s) \\ -1 & \text { if } h \in \Delta^{-}(s) \\ 0 & \text { otherwise. }\end{cases}
$$

Problem $P$ was considered in [27], [28] and is closely related to the uncontrollable flow raised by Iri $[18,19]$. It arises from the following situation. Considering the maximum flow problem, we usually take it for granted that each arc flow is controllable, i.e., we can freely increase and decrease it as long as the conservation equations and capacity constraints are kept satisfied. However, in the situation where we are not able or allowed to reduce the given arc flow, we may fail to reach a maximum flow and get stuck in an undesired maximal flow. With such restricted controllability, we may end up with different maximal flows depending on the initial flow as well as the way of augmentation. Therefore the minimum of the flow values that are attained by maximal flows will play a prominent role in evaluating how inefficiently the network can be utilized. Note that the problem encompasses the minimum maximal matching problem, which is known to be $N P$-hard, e.g., [14].

Since the set $X_{M}$ is in general nonconvex, Problem $P$ is one of the typical multiextremal optimization problems. See e.g., Horst, Pardalos and Thoai [15] and Horst and Tuy [17]. Actually, the set $X_{M}$ can be considered as the set of all efficient solutions of the multiple objective programming problem (vector optimization problem)

$$
\begin{array}{l|ll}
M O & \text { vector } \max & x \\
\text { s.t. } & x \in X,
\end{array}
$$

so that Problem $P$ is a special case of the class of optimization problems over an efficient set. Solution methods for optimization problems over an efficient set can be found e.g., in Lethi, Pham and Muu [2], Benson [4, 5], Benson and Lee [7], Benson and Sayin [8], Dauer and Fosnaugh [11], Horst and Thoai [16], Muu [20], Sayin [25], Thoai [32, 33], Thach, Konno and Yokota [30], White [35], Yamada, Tanino and Inuiguchi [36], Phong and Tuyen [23] and Yamamoto [37]. A common feature of these methods is, however, that they can be only successfully applied to problems where the underlying multiple objective programming problem has a small number of objective functions.

In the present article, we first formulate the underlying problem equivalently as a linear program with an additional nonconvex constraint, and then propose a method to combine local and global optimization techniques for solving the resulting problem in a way that the advantageous network problem structure will be successfully applied.

The equivalent formulation of Problem $P$ with its advantageous network flow structure is discussed in the next section. Suitable linear program relaxations of this equivalent problem is established in Section 3. Section 4 presents different local and global optimization techniques which are used for the establishment of the 
combination algorithm in Section 5. The convergence of the combination algorithm depends on the branching procedure within a branch and bound scheme for checking global optimality. The case of finite convergence is discussed in Section 4 while establishing the branch and bound method using integral rectangular division for the branching procedure. For the use of simplicial branch and bound procedure, some kind of approximate optimal solutions is introduced in Section 5 so that the combination algorithm yields an approximate optimal solution after finitely many iterations. The final section contains some conclusions.

\section{Equivalent Problem Formulations}

In this paper we denote by $\mathbb{R}^{k}$ and $\mathbb{R}_{k}$ the set of $k$-dimensional column vectors and the set of $k$-dimensional row vectors, respectively. As mentioned in the previous section, the set $X_{M}$ of maximal flows is exactly the efficient set of $M O$. From well-known results in multiple objective programming, e.g., Benson [6], Sawaragi, Nakayama and Tanino [26], Steuer [29] and White [34], there is a compact subset, say $\Lambda$, of $\mathbb{R}_{n++}=\left\{\lambda \mid \lambda \in \mathbb{R}_{n} ; \lambda>0\right\}$ such that a point $x$ belongs to $X_{M}$ if and only if it maximizes $\lambda x$ over $X$ for some $\lambda \in \Lambda$, i.e.,

$$
X_{M}=\{x \mid x \in X ; \lambda x \geq \phi(\lambda) \text { for some } \lambda \in \Lambda\},
$$

where

$$
\phi(\lambda)=\max \{\lambda y \mid y \in X\} .
$$

In what follows we denote by $e$ the row vector of ones and by $\mathbb{Z}_{n}$ the set of $n$ dimensional integral row vectors. The following theorem shows that a finite set of integral points of $\mathbb{R}_{n++}$ suffices as $\Lambda$.

Theorem 2.1. $\Lambda_{1}=\left\{\lambda \mid \lambda \in \mathbb{Z}_{n} ; e \leq \lambda \leq n e\right\}$ suffices as $\Lambda$ in (2.1).

To prove the above theorem we need the following lemmas.

Let $\bar{x} \in \mathbb{R}^{n}$ be a given maximal flow. Further let $F$ be the index set defined by $F=\left\{h \mid h \in E ; \bar{x}_{h}=c_{h}\right\}$ and $\bar{F}=E \backslash F$. Note that $F \neq \emptyset$. We refer to a directed path from node $i$ to node $j$ as an $i-j$ path.

Lemma 2.2. Let $G$ be the graph of node set $V$ and arc set $\bar{F}$.

(i) $G$ is acyclic and does not contain an $s-t$ path or a $t-s$ path.

(ii) For each node $i \in V \backslash\{s, t\}$ at least one of the following two cases occurs:

case 1: $G$ has neither an $s-i$ path nor a $t-i$ path.

case 2: $G$ has neither an $i-s$ path nor an $i-t$ path.

Proof. The assertion (i) is clear from the fact that $\bar{x}$ is a maximal flow. Let $i$ be an arbitrary node and suppose that case 1 of (i) does not occur, i.e., there is either an $s-i$ path or a $t-i$ path. If there is an $s-i$ path, we have by (i) that there is neither an $i-s$ path nor an $i-t$ path, and if there is a $t-i$ path, we see that there is neither an $i-s$ path nor an $i-t$ path. These correspond to case 2 .

Next let $a_{\ell}$ denote the row of the incidence matrix $A$ of the network corresponding to node $\ell \in V \backslash\{s, t\}$. Suppose we are given a nonempty subset $U$ of $V \backslash\{s, t\}$ and 
let

$$
\begin{aligned}
& \Delta_{E}^{+}(U)=\{h \mid h=(i, j) \in E ; i \in U ; j \in V \backslash U\} \\
& \Delta_{E}^{-}(U)=\{h \mid h=(i, j) \in E ; j \in U ; i \in V \backslash U\} .
\end{aligned}
$$

Then it will be readily seen from the definition of the incidence matrix that

$$
\sum_{\ell \in U} a_{\ell}=\sum_{k \in \Delta_{E}^{+}(U)} e_{k}+\sum_{k \in \Delta_{E}^{-}(U)}\left(-e_{k}\right) .
$$

Lemma 2.3. For each $h \in \bar{F}$ it holds that

$$
e_{h}=\alpha_{h} \sum_{\ell \in V_{h}} a_{\ell}+\sum_{k \in F} \beta_{h k} e_{k}-\sum_{k \in E \backslash\{h\}} \gamma_{h k} e_{k}
$$

for some $\alpha_{h} \in\{-1,1\}, V_{h} \subseteq V \backslash\{s, t\}, \beta_{h k} \in\{0,1\}$ and $\gamma_{h k} \in\{0,1\}$.

Proof. Let $h=(i, j)$ and consider the following two cases.

case 1: node $i$ satisfies the condition of case 1 of Lemma 2.2.

Let

$$
V_{h}^{+}=\{\ell \mid \ell \in V ; \text { there is an } \ell-i \text { path of } G\} .
$$

Then we see from Lemma 2.2 that $s, t, j \notin V_{h}^{+}$and that no arcs of $\bar{F}$ come into $V_{h}^{+}$ from its complement $\overline{V_{h}^{+}}=V \backslash V_{h}^{+}$. Therefore the cut $\left(V_{h}^{+}, \overline{V_{h}^{+}}\right)$consists of the three sets of arcs: $\Delta_{\bar{F}}^{+}\left(V_{h}^{+}\right), \Delta_{F}^{+}\left(V_{h}^{+}\right)$and $\Delta_{F}^{-}\left(V_{h}^{+}\right)$. By $(2.5)$ we obtain

$$
\sum_{\ell \in V_{h}^{+}} a_{\ell}=\sum_{k \in \Delta_{F}^{+}\left(V_{h}^{+}\right)} e_{k}+\sum_{k \in \Delta_{F}^{+}\left(V_{h}^{+}\right)} e_{k}+\sum_{k \in \Delta_{F}^{-}\left(V_{h}^{+}\right)}\left(-e_{k}\right)
$$

which is rewritten as, since $h \in \Delta_{\bar{F}}^{+}\left(V_{h}^{+}\right)$,

$$
\sum_{\ell \in V_{h}^{+}} a_{\ell}=e_{h}+\sum_{k \in \Delta_{\frac{+}{F}}\left(V_{h}^{+}\right) \backslash\{h\}} e_{k}+\sum_{k \in \Delta_{F}^{+}\left(V_{h}^{+}\right)} e_{k}+\sum_{k \in \Delta_{F}^{-}\left(V_{h}^{+}\right)}\left(-e_{k}\right),
$$

Thus we obtain

$$
e_{h}=\sum_{\ell \in V_{h}^{+}} a_{\ell}+\sum_{k \in \Delta_{F}^{-}\left(V_{h}^{+}\right)} e_{k}-\left(\sum_{k \in \Delta_{F}^{+}\left(V_{h}^{+}\right)} e_{k}+\sum_{k \in \Delta_{\frac{+}{F}}^{+}\left(V_{h}^{+}\right) \backslash\{h\}} e_{k}\right) .
$$

case 2: node $i$ satisfies the condition of case 2 of Lemma 2.2.

Since node $i$ satisfies the conditon of case 2 and arc $h=(i, j)$ is in $\bar{F}$, node $j$ also satisfies that condition. Let $V_{h}^{-}=\{\ell \mid \ell \in V$; there is a $j-\ell$ path of $G\}$. Then we see $s, t, i \notin V_{h}^{-}$and that no $\operatorname{arcs}$ of $\bar{F}$ go from $V_{h}^{-}$into $\overline{V_{h}^{-}}=V \backslash V_{h}^{-}$, and the cut $\left(V_{h}^{-}, \overline{V_{h}^{-}}\right)$consists of $\Delta_{\bar{F}}^{-}\left(V_{h}^{-}\right), \Delta_{F}^{-}\left(V_{h}^{-}\right)$and $\Delta_{F}^{+}\left(V_{h}^{-}\right)$. Therefore

$$
\begin{aligned}
\sum_{\ell \in V_{h}^{-}} a_{\ell} & =\sum_{k \in \Delta_{\bar{F}}^{-}\left(V_{h}^{-}\right)}\left(-e_{k}\right)+\sum_{k \in \Delta_{F}^{-}\left(V_{h}^{-}\right)}\left(-e_{k}\right)+\sum_{k \in \Delta_{F}^{+}\left(V_{h}^{-}\right)} e_{k} \\
& =-e_{h}+\sum_{k \in \Delta_{\bar{F}}^{-}\left(V_{h}^{-}\right) \backslash\{h\}}\left(-e_{k}\right)+\sum_{k \in \Delta_{F}^{-}\left(V_{h}^{-}\right)}\left(-e_{k}\right)+\sum_{k \in \Delta_{F}^{+}\left(V_{h}^{-}\right)} e_{k} .
\end{aligned}
$$


Hence

$$
e_{h}=\left(-\sum_{\ell \in V_{h}^{-}} a_{\ell}\right)+\sum_{k \in \Delta_{F}^{+}\left(V_{h}^{-}\right)} e_{k}-\left(\sum_{k \in \Delta_{F}^{-}\left(V_{h}^{-}\right)} e_{k}+\sum_{k \in \Delta_{\bar{F}}^{-}\left(V_{h}^{-}\right) \backslash\{h\}} e_{k}\right) .
$$

This completes the proof.

Proof of Theorem 2.1.

By Lemma 2.3 we see for each $h \in \bar{F}$

$$
e_{h}+\sum_{k \in E \backslash\{h\}} \gamma_{h k} e_{k}=\alpha_{h} \sum_{\ell \in V_{h}} a_{\ell}+\sum_{k \in F} \beta_{h k} e_{k}
$$

for some $\alpha_{h} \in\{-1,1\}, V_{h} \subseteq V \backslash\{s, t\}, \beta_{h k} \in\{0,1\}$ and $\gamma_{h k} \in\{0,1\}$. Adding these equations over $h \in \bar{F}$ and the identities $e_{h}=e_{h}$ for $h \in F$, we obtain

$$
\sum_{k \in E} \lambda_{k} e_{k}=\sum_{\ell \in V \backslash\{s, t\}} \delta_{\ell} a_{\ell}+\sum_{k \in F} \zeta_{k} e_{k},
$$

where $\lambda_{k}=1+\sum_{h \in E \backslash\{k\}} \gamma_{h k}$ for $k \in E, \zeta_{k}=\sum_{h \in F} \beta_{h k}+1$ for $k \in F$, and $\delta_{\ell}$ is appropriately defined for $\ell \in V \backslash\{s, t\}$. Note that

$$
1 \leq \lambda_{k} \leq 1+(n-1)=n
$$

for $k \in E$ and $\zeta_{k} \geq 0$ for $k \in F$. Let $\lambda=\sum_{k \in E} \lambda_{k} e_{k}$. Clearly, $\lambda \in \Lambda_{1}$. Then for any feasible flow $x$ it holds that

$$
\begin{aligned}
\lambda \bar{x} & =\sum_{k \in E} \lambda_{k} e_{k} \bar{x}=\sum_{\ell \in V \backslash\{s, t\}} \delta_{\ell} a_{\ell} \bar{x}+\sum_{k \in F} \zeta_{k} e_{k} \bar{x} \\
& =\sum_{k \in F} \zeta_{k} \bar{x}_{k}=\sum_{k \in F} \zeta_{k} c_{k} \\
& \geq \sum_{k \in F} \zeta_{k} x_{k}=\sum_{\ell \in V \backslash\{s, t\}} \delta_{\ell} a_{\ell} x+\sum_{k \in F} \zeta_{k} e_{k} x=\lambda x,
\end{aligned}
$$

meaning that the maximal flow $\bar{x}$ maximizes $\lambda x$ over the set of feasible flows.

By the porperty that $\phi(\alpha \lambda)=\alpha \phi(\lambda)$ for $\alpha>0$, any compact subset of $\mathbb{R}_{n++}$ whose conical hull contains the conical hull of $\Lambda_{1}$ works as $\Lambda$. Therefore we could replace $\Lambda$ by the simplex

$$
\Lambda_{2}=\left\{\lambda \mid \lambda \in \mathbb{R}_{n} ; \lambda \geq e ; \sum_{i=1}^{n} \lambda_{i}=M\right\}
$$

if $M$ is sufficiently large.

Corollary 2.4. $n^{2}$ suffices for $M$ defining $\Lambda_{2}$ of (2.20).

Proof. Let $\bar{x}$ be a maximal flow. By Theorem 2.1 it maximizes $\lambda x$ over the feasible flows for some $\lambda \in \mathbb{R}_{n}$ such that $1 \leq \lambda_{h} \leq n$ for each $h \in E$. Let $\bar{\lambda}=$ $\left(n^{2} / \sum_{h \in E} \lambda_{h}\right) \lambda$. Then since $n^{2} \geq \sum_{h \in E} \lambda_{h}, \bar{\lambda}$ lies in $\Lambda_{2}$ defined for $M=n^{2}$ and $\bar{x}$ maximizes $\bar{\lambda} x$ over the feasible flows.

Theorem 2.1 implies that Problem $P$ can be solved in theory by the following method. For each integral vector $\lambda \in \Lambda_{1}$ identify the optimal face $F(\lambda)$ of $\max \{\lambda x \mid x \in X\}$, and solve $\min \{d x \mid x \in F(\lambda)\}$ for a solution $x(\lambda)$. Note that any point of $F(\lambda)$ is a maximal flow, and it is readily seen that $x\left(\lambda^{*}\right)$ such that 
$d x\left(\lambda^{*}\right)=\min \left\{d x(\lambda) \mid \lambda \in \Lambda_{1}\right\}$ is a solution of $P$. However, the integral points to be considered amount to $n^{n}$, so that this method is not practical.

Remark 2.5. The optimal face $F(\lambda)$ is an outer semicontinuous mapping when considered as a point to set mapping, i.e., for a sequcence $\left\{\lambda_{\nu}\right\}$ converging to $\lambda$ any cluster point of the sequence $\left\{x^{\nu}\right\}$ with $x^{\nu} \in F\left(\lambda_{\nu}\right)$ is contained in $F(\lambda)$. See Exercise 1.19 of Rockafellar and Wets [24]. Then $d x(\lambda)$ is a lower semicontinuous function in $\lambda$, i.e., $\liminf _{\nu} d x\left(\lambda_{\nu}\right) \geq d x(\lambda)$ for any sequence $\left\{\lambda_{\nu}\right\}$ converging to $\lambda$. Therefore for a given $\epsilon>0$ each $\lambda$ has a neighborhood such that $d x\left(\lambda^{\prime}\right) \geq d x(\lambda)-\epsilon$ holds for any $\lambda^{\prime}$ in the neighborhood. This means that it is very likely that $\lambda$ 's with large objective function values $d x(\lambda)$ make a cluster. Thus the divide and conquer principle or the branch and bound method should work efficiently.

The above arguments yield two different representations of the set $X_{M}$ of maximal flows:

$$
\begin{aligned}
X_{M} & =\left\{x \mid x \in X ; \lambda x \geq \phi(\lambda) \text { for some } \lambda \in \Lambda_{1}\right\} \\
& =\left\{x \mid x \in X ; \lambda x \geq \phi(\lambda) \text { for some } \lambda \in \Lambda_{2}\right\},
\end{aligned}
$$

each of which will in the following sections provide a scheme for solving the problem.

Now Problem $P$ is written equivalently as

$$
\begin{array}{l|ll}
P & \begin{array}{ll}
\min & d x \\
\text { s.t. } & \lambda x-\phi(\lambda) \geq 0 \\
& \lambda \in \Lambda,
\end{array}
\end{array}
$$

where $\Lambda$ is either $\Lambda_{1}$ or $\Lambda_{2}$ with $M=n^{2}$.

Lemma 2.6. Assume that each arc capacity $c_{h}$ is a nonnegative integer. Let $X_{V}$ denote the set of vertices of the polytope $X$, and let $\bar{x} \in X_{V} \cap X_{M}$. Then, whenever $\left\{x \mid x \in X_{M} ; d x<d \bar{x}\right\} \neq \emptyset$, there exists $x \in X_{M} \cap X_{V}$ such that $d x \leq d \bar{x}-1$.

Proof. Note first that $d$ is an integral vector. Then this lemma is a direct consequence of the two well-known facts that each vertex of $X$ is an integral vector and that Problem $P$ has an optimal solution in the vertex set of $X$.

\section{Linear Program Relaxation}

In this section we explain a linear program relaxation of Problem $P$. For the sake of further argument, we consider the following problem with $\lambda$ restricted to a polytope, say $S$, contained in $\Lambda$ :

$$
\begin{aligned}
& \begin{array}{ll}
\min & d x \\
\text { s.t. } & A x=0
\end{array} \\
& 0 \leq x \leq c \\
& \lambda x-\phi(\lambda) \geq 0 \\
& \lambda \in S \text {. }
\end{aligned}
$$

Let

$$
\Phi(S)=\min \{\phi(\lambda) \mid \lambda \in S\},
$$


where the function $\phi(\lambda)$ is defined in (2.2). Our method for constructing linear program relaxations of the nonlinear, nonconvex programming problem $P(S)$ is based on the following result.

Lemma 3.1. Let $\left\{\lambda_{1}, \cdots, \lambda_{q}\right\}$ be the vertex set of $S$. Then Problem $P(S)$ is relaxed to the following linear program in variables $x^{\ell} \in \mathbb{R}^{n}$ for $\ell=1, \cdots, q$ :

$$
\bar{P}(S) \quad \mid \begin{array}{ll}
\min & \sum_{\ell=1}^{q} d x^{\ell} \\
\text { s.t. } & A x^{\ell}=0 \text { for } \ell=1, \ldots, q \\
& x^{\ell} \geq 0 \text { for } \ell=1, \ldots, q \\
& \sum_{\ell=1}^{q} x^{\ell} \leq c \\
& \sum_{\ell=1}^{q} \lambda_{\ell} x^{\ell}-\Phi(S) \geq 0,
\end{array}
$$

i.e., the optimal value $\bar{\mu}(S)$ of Problem $\bar{P}(S)$ yields a lower bound of the optimal value of Problem $P(S)$.

Proof. We show that for any feasible solution $(x, \lambda)$ of Problem $P(S)$ there exists a feasible solution $\left(x^{1}, \cdots, x^{q}\right)$ of Problem $\bar{P}(S)$ satisfying

$$
d x=\sum_{\ell=1}^{q} d x^{\ell} .
$$

Since $\lambda \in S$,

$$
\lambda=\sum_{\ell=1}^{q} \beta_{\ell} \lambda_{\ell}
$$

for some nonnegative numbers $\beta_{\ell}(\ell=1, \ldots, q)$ such that $\sum_{\ell=1}^{q} \beta_{\ell}=1$. Define

$$
x^{\ell}=\beta_{\ell} x \text { for } \ell=1, \cdots, q .
$$

Then clearly $\sum_{\ell=1}^{q} x^{\ell}=x$ and $\left(x^{1}, \ldots, x^{q}\right)$ satisfies the first three constraints of $\bar{P}(S)$. For the last constraint we have

$$
\sum_{\ell=1}^{q} \lambda_{\ell} x^{\ell}=\sum_{\ell=1}^{q} \lambda_{\ell} \beta_{\ell} x=\lambda x \geq \phi(\lambda) \geq \Phi(S),
$$

where the last two inequalities follow from the assumption that $(x, \lambda)$ is feasible to $P(S)$ and from the definition of $\Phi(S)$ in (3.1).

Remark 3.2. If Problem $\bar{P}(S)$ is infeasible, so is Problem $P(S)$. In this case we set $\bar{\mu}(S)=+\infty$.

Remark 3.3. The value $\Phi(S)$ is determined in two different ways, both of which come from the bilinearlity of $\lambda x$. The first way is

$$
\begin{aligned}
\Phi(S) & =\min _{\lambda \in S} \max _{x \in X} \lambda x \\
& =\min \{r \mid \lambda \in S ; r \geq \lambda x \text { for all } x \in X\} \\
& =\min \left\{r \mid \lambda \in S ; r \geq \lambda x^{\ell} \text { for all } x^{\ell} \in X_{V}\right\} .
\end{aligned}
$$


Note that we need not know all vertices of $X$ in advance if we use the so-called column generation technique in soving this problem. Applying the well-known minimax theorem yields the second way:

$$
\begin{aligned}
\Phi(S) & =\min _{\lambda \in S} \max _{x \in X} \lambda x \\
& =\max _{x \in X} \min _{\lambda \in S} \lambda x \\
& =\max \{r \mid x \in X ; r \leq \lambda x \text { for all } \lambda \in S\} \\
& =\max \left\{r \mid x \in X ; r \leq \lambda_{\ell} x \text { for } \ell=1, \ldots, q\right\} .
\end{aligned}
$$

In either way $\Phi(S)$ is obtained by solving a linear programming.

Remark 3.4. Because of the constraint $\sum_{\ell=1}^{q} \lambda_{\ell} x^{\ell}-\Phi(S) \geq 0$, the advantageous network structure of Problem $\bar{P}(S)$ may be violated. To overcome this possible difficulty, we propose to use here the Lagrangian relaxation of this problem.

Consider the Lagrangian relaxation problem with a multiplier $\pi \geq 0$

$$
\begin{array}{|ll}
\min & \sum_{\ell=1}^{q} d x^{\ell}+\pi\left(\Phi(S)-\sum_{\ell=1}^{q} \lambda_{\ell} x^{\ell}\right) \\
\text { s.t. } & A x^{\ell}=0 \text { for } \ell=1, \ldots, q \\
& x^{\ell} \geq 0 \text { for } \ell=1, \ldots, q \\
& \sum_{\ell=1}^{q} x^{\ell} \leq c .
\end{array}
$$

For each $j=1,2, \ldots, n$ let

$$
\lambda_{j}^{\max }=\max \left\{\left(\lambda_{\ell}\right)_{j} \mid \ell=1, \ldots, q\right\}
$$

and let $d^{\prime}(\pi) \in \mathbb{R}_{n}$ be the vector whose $j$ th component $d_{j}^{\prime}(\pi)$ is defined by

$$
d_{j}^{\prime}(\pi)=d_{j}-\pi \lambda_{j}^{\max }
$$

Then clearly for a fesible solution $\left(x^{1}, \ldots, x^{q}\right)$

$$
\sum_{\ell=1}^{q} d^{\prime}(\pi) x^{\ell} \leq \sum_{\ell=1}^{q}\left(d-\pi \lambda_{\ell}\right) x^{\ell},
$$

so that the above Lagrangian relaxation can be relaxed further by the problem

$$
\begin{array}{|ll}
\min & \sum_{\ell=1}^{q} d^{\prime}(\pi) x^{\ell}+\pi \Phi(S) \\
\text { s.t. } & A x^{\ell}=0 \text { for } \ell=1, \ldots, q \\
& x^{\ell} \geq 0 \text { for } \ell=1, \ldots, q \\
& \sum_{\ell=1}^{q} x^{\ell} \leq c,
\end{array}
$$

which is actually equivalent to the following minimum cost network flow problem in $n$ variables:

$$
\begin{array}{ll}
\min & d^{\prime}(\pi) x+\pi \Phi(S) \\
\text { s.t. } & A x=0 \\
& 0 \leq x \leq c
\end{array}
$$


An efficient choice for the multiplier $\pi$ can be taken using the well-known parametric linear programming technique. See for example Ahuja, Magnanti and Orlin [1], Belling-Seib, Mevet and Muller [3] and Bryson [10].

The dual problem of $\bar{P}(S)$, denoted by $\bar{D}(S)$, is written as

$$
\begin{array}{l|ll}
\max & -v c+z \Phi(S) \\
& \text { s.t. } & u_{\ell} A-v+z \lambda_{\ell} \leq d \text { for } \ell=1, \ldots, q \\
& (v, z) \geq 0,
\end{array}
$$

where $u_{k} \in \mathbb{R}_{m}, v \in \mathbb{R}_{n}, z \in \mathbb{R}$.

Lemma 3.5. A nonnegative vector $(v, z)$ satisfies the constraints of $\bar{D}(S)$ together with some $u_{1}, \ldots, u_{q} \in \mathbb{R}_{m}$ if and only if for any $\lambda \in S$ there is $u_{\lambda} \in \mathbb{R}_{m}$ such that $u_{\lambda} A-v+z \lambda \leq d$.

Proof. The "if" part is readily seen by setting $u_{\ell}=u_{\lambda_{\ell}}$ for $\ell=1, \ldots, q$. To show the "only if" part, let $\lambda$ be a given vector of $S$. Then $\lambda=\sum_{\ell=1}^{q} \beta_{\ell} \lambda_{\ell}$ for some nonnegative $\beta_{\ell}$ 's with $\sum_{\ell=1}^{q} \beta_{\ell}=1$. Let $u_{\lambda}=\sum_{\ell=1}^{q} \beta_{\ell} u_{\ell}$. Then

$$
\begin{aligned}
u_{\lambda} A-v+z \lambda & =\left(\sum_{\ell=1}^{q} \beta_{\ell} u_{\ell}\right) A-v+z\left(\sum_{\ell=1}^{q} \beta_{\ell} \lambda_{\ell}\right) \\
& =\sum_{\ell=1}^{q} \beta_{\ell}\left(u_{\ell} A-v+z \lambda_{\ell}\right) \leq d .
\end{aligned}
$$

The lower bound $\bar{\mu}(S)$ has the following properties, which will be utilized within the branch and bound procedure.

Lemma 3.6. Suppose $\emptyset \neq S_{2} \subseteq S_{1} \subseteq \Lambda$. Then

$$
-\infty<\bar{\mu}\left(S_{1}\right) \leq \bar{\mu}\left(S_{2}\right) .
$$

Proof. Suppose $(v, z)$ is a feasible solution of $\bar{D}\left(S_{1}\right)$ together with $\left(u_{1}, \ldots, u_{q}\right)$. Then by Lemma 3.5 , we see that $(v, w, z)$ is a feasible solution of $\bar{D}\left(S_{2}\right)$ with some $\left(u_{1}^{\prime}, \ldots, u_{q}^{\prime}\right)$. Since the objective function value of $\bar{D}(S)$ is determined solely by $(v, z)$, we see the monotonicity $\bar{\mu}\left(S_{1}\right) \leq \bar{\mu}\left(S_{2}\right)$. The fact that $-\infty<\bar{\mu}\left(S_{1}\right)$ is a direct consequence of the boundedness of the feasible region of $\bar{P}\left(S_{1}\right)$.

We now show that the relaxation problem $\bar{P}(S)$ can be substantially simplified when $S$ is a hyper rectangle.

Lemma 3.7. If $\lambda \leq \lambda^{\prime}$, then $\phi(\lambda) \leq \phi\left(\lambda^{\prime}\right)$.

Proof. Let $\bar{x}$ be a point of $X$ which maximizes $\lambda x$ over $X$. Since $\bar{x} \geq 0$ we have $\left(\lambda-\lambda^{\prime}\right) \bar{x} \leq 0$, and hence

$$
\phi(\lambda)=\lambda \bar{x} \leq \lambda^{\prime} \bar{x} \leq \phi\left(\lambda^{\prime}\right) .
$$


Lemma 3.8. Let $\left\{\lambda_{1}, \ldots, \lambda_{q}\right\}$ be the vertex set of $S \subseteq \Lambda$ and suppose $\lambda_{1} \geq \lambda_{\ell} \geq \lambda_{q}$ for all $\ell=1, \ldots, q$. Then Problem $\bar{P}(S)$ is equivalent to the problem

$\bar{P}^{\prime}(S) \quad \mid \begin{array}{ll}\min \quad & d x \\ \text { s.t. } & A x=0 \\ & 0 \leq x \leq c \\ & \lambda_{1} x-\phi\left(\lambda_{q}\right) \geq 0\end{array}$

in $n$ variables.

Proof. Note that $\lambda_{q} \leq \lambda_{\ell}$ for all $\ell=1, \ldots, q$ implies that $\lambda_{q} \leq \lambda$ for all $\lambda \in S$. Then by Lemma 3.7 we have $\Phi(S)=\phi\left(\lambda_{q}\right)$.

Let $\left(x^{1}, \ldots, x^{q}\right)$ be a feasible solution of $\bar{P}(S)$ and let $x=\sum_{\ell=1}^{q} x^{\ell}$. Then clearly $A x=0$ and $0 \leq x \leq c$. Furthermore, since $x^{\ell} \geq 0$ we obtain

$$
\begin{gathered}
\lambda_{1} x=\lambda_{1} \sum_{\ell=1}^{q} x^{\ell}=\sum_{\ell=1}^{q} \lambda_{1} x^{\ell} \geq \sum_{\ell=1}^{q} \lambda_{\ell} x^{\ell} \geq \Phi(S) \\
d x=\sum_{\ell=1}^{q} d x^{\ell} .
\end{gathered}
$$

This means that $\bar{\mu}(S) \geq \bar{\mu}^{\prime}(S)$, where $\bar{\mu}^{\prime}(S)$ is the optimal objective function value of $\bar{P}^{\prime}(S)$. Let $x$ be a feasible solution of $\bar{P}^{\prime}(S)$ and let $x^{1}=x, x^{2}=\cdots=x^{q}=0$. Then $\left(x^{1}, \ldots, x^{q}\right)$ clearly satisfies the constraints of $\bar{P}(S)$, meaning $\bar{\mu}(S) \leq \bar{\mu}^{\prime}(S)$.

From Lemma 3.8 if we take $\Lambda_{1}$ as $\Lambda$ and consider a hyper rectangle $S=\{\lambda \mid$ $\left.\lambda_{q} \leq \lambda \leq \lambda_{1}\right\}$ contained in $\Lambda_{1}$, then, although $S$ has as many as $2^{n}$ vertices, the relaxation problem $\bar{P}^{\prime}(S)$ has only $n$ variables as Problem $P(S)$ does. This might be an advantage of using $\Lambda_{1}$.

\section{Local Search and Checking up Global Optimality}

4.1. Local Search. The algorithms for the optimization over the efficient set proposed by Philip [22], Ecker and Song [12], Fülöp [13] and Bolintineanu [9] are mainly based on the technique of moving from an efficient vertex to an efficient neighbor with a smaller objective function value via an efficient edge. In this section, following their argment we will explain a local search technique calledAdjacent Vertex Search Procedure.

We say that a maximal flow is extreme if it is a vertex of the set of feasible flows $X$. It is known, e.g. Naccache [21], Sawaragi, Nakayama and Tanino [26] and White [34], the set of maximal flows $X_{M}$ is connected, and all extreme maximal flows are conneced by paths of edges consisting of maximal flows. Thus, starting from an extreme maximal flow, we could reach an optimal solution of Problem $P$ by a series of pivot operations in theory. However, we cannot decrease the objective function value monotonically along the path that we trace, i.e., we might be eventually caught by a non-optimal extreme maximal flow none of whose neighboring extreme maximal flows have a smaller objective function value. When this occurs, it is a local minimum solution. See for example Bolintineanu [9]. 
The Adjacent Vertex Search $(A V S)$ Procedure goes as follows. We denote by $\left[x, x^{\prime}\right]$ the edge of $X$ connecting $x$ and $x^{\prime}$ for $x, x^{\prime} \in X_{V}$ and

$$
N_{M}(x)=\left\{x^{\prime} \mid x^{\prime} \in X_{V} \cap X_{M} ;\left[x, x^{\prime}\right] \subseteq X_{M}\right\} .
$$

\section{Adjacent Vertex Search (AVS) Procedure}

$\langle\langle$ Initialization $\rangle\rangle$

Find $x^{0} \in X_{V} \cap X_{M}$. If $N_{M}\left(x^{0}\right)=\emptyset$, then $x^{0}$ is an optimum solution of $P$. Otherwise, set $k \leftarrow 0$ and go to Step $k$.

$\langle\langle$ Step $k\rangle\rangle$

$\langle k 1\rangle$ If $\left\{x \mid x \in N_{M}\left(x^{k}\right) ; d x<d x^{k}\right\} \neq \emptyset$, choose $x^{k+1}$ from this set, let $k \leftarrow k+1$ and go to Step $k$.

$\langle k 2\rangle$ Otherwise, set $v \leftarrow x^{k}$ and stop.

Note that the initial extreme maximal flow $x^{0}$ is easily found by choosing an arbitrary positive vector $\lambda$ and maximizing $\lambda x$ over $X$. The AVS Procedure generates a sequence of distinct extreme maximal flows $x^{0}, x^{1}, \ldots, x^{k}$ with decreasing objective funtion values, which implies owing to the integrality property that $d x^{k} \leq d x^{0}-k$.

4.2. Checking up Global Optimality. We present in this subsection two Branch and Bound (BB) Procedures for handling the following problem:

$$
C G O(\alpha)
$$

For a given integer $\alpha$, find an extreme maximal flow $v \in X_{M} \cap X_{V}$ such that $d v \leq \alpha$, or show that there does not exist such a point.

As shown in Section 2, for the description of the set $X_{M}$, we can use one of two sets $\Lambda_{1}$ and $\Lambda_{2}$. The set $\Lambda_{1}$ defined in Theorem 2.1 consists of all integral vectors contained in the rectangle $\{\lambda: e \leq \lambda \leq n e\}$, while the set $\Lambda_{2}$ is an $(n-1)$-simplex defined in (2.20). Before presenting two branch and bound procedures for handling Problem $C G O(\alpha)$ according to $\Lambda_{1}$ and $\Lambda_{2}$, respectively, we propose here two kinds of polyhedral partitions called integral rectangular division and simplicial division.

\section{Integral Rectangular Division}

Let $S \subset \Lambda_{1}$ be a rectangle with integral bound vectors, which contains more than one integral vector and is defined by

$$
S=\left\{x \mid x \in \mathbb{R}^{n} ; a_{j} \leq x_{j} \leq b_{j},(j=1, \cdots, n)\right\}=\left\{x \mid x \in \mathbb{R}^{n} ; a \leq x \leq b\right\},
$$

where $a_{j}, b_{j}$ are integers satisfying $a_{j} \leq b_{j}(j=1, \cdots, n)$. Further, let $S_{1}, \cdots, S_{q}$ be rectangles with integral bound vectors having the following properties

$$
\begin{aligned}
& \bigcup_{i=1}^{q} S_{i} \subset S, \\
& S_{i} \cap S_{j}=\emptyset \text { for } i \neq j, \\
& \bigcup_{i=1}^{q}\left(S_{i} \cap \mathbb{Z}_{n}\right)=S \cap \mathbb{Z}_{n} .
\end{aligned}
$$


Then we say that $\left\{S_{1}, \cdots, S_{q}\right\}$ is an integral rectangular division of the rectangle $S$.

As a special case of this division, we consider the following integral rectangular bisection.

Let $u \in S$ such that $u \neq b$, and let $\ell \in\{1, \cdots, n\}$ such that $u_{\ell}<b_{\ell}$, (note that such an index $\ell$ exists, whenever $S$ contains more than one integral vector). For each real number $t$, denoting by $\lfloor t\rfloor$ the largest integer which is less than or equal to $t$, we define two rectangles

$$
\begin{aligned}
& S_{1}=\left\{x \mid x \in S ; x_{\ell} \leq\left\lfloor u_{\ell}\right\rfloor\right\} \\
& S_{2}=\left\{x \mid x \in S ; x_{\ell} \geq\left\lfloor u_{\ell}\right\rfloor+1\right\} .
\end{aligned}
$$

Then, it is easy to verify that $\left\{S_{1}, S_{2}\right\}$ is an integral rectangular division of $S$. We say that $S$ is divided into $\left\{S_{1}, S_{2}\right\}$ by an integral rectangular bisection using the point $u$.

\section{Simplicial Division}

Let $S$ be an $(n-1)$-simplex with vertex set $S_{V}=\left\{\lambda_{1}, \ldots, \lambda_{n}\right\}$. Choose a point $\bar{\lambda} \in S \backslash S_{V}$ which is uniquely represented as

$$
\bar{\lambda}=\sum_{i=1}^{n} \beta_{i} \lambda_{i}, \beta_{i} \geq 0 \quad(i=1, \ldots, n), \quad \sum_{i=1}^{n} \beta_{i}=1,
$$

and for each $i$ such that $\beta_{i}>0$ form the simplex $S_{i}$ obtained from $S$ by replacing the vertex $\lambda_{i}$ by $\bar{\lambda}$, i.e., $S_{i}=\operatorname{co}\left\{\lambda_{1}, \ldots, \lambda_{i-1}, \bar{\lambda}, \lambda_{i+1}, \ldots, \lambda_{n}\right\}$, where $\operatorname{co} A$ denotes the convex hull of a set $A$. This division is called a radial simplicial division.

When $\bar{\lambda}$ is the midpoint of a longest edge of $S$, then we obtain two subsimplices. This special case is called a simplicial bisection.

As discussed in the preceding section, our BB Procedures are based on the linear relaxation $\bar{P}(S)$ of the subproblem $P(S)$ with $\lambda$ restricted to a subset $S \subseteq \Lambda$, where $\Lambda$ is either $\Lambda_{1}$ or $\Lambda_{2}$. The branching process subdivides $\Lambda$ into finitely many subsets yielding a class of subproblems to be solved. In the algorithm to be proposed we repeatedly apply the AVS Procedure, which provides a local minimum incumbent solution $v^{\nu}$, and then one of the BB Procedures to check up the global optimality of $v^{\nu}$. The chosen BB procedure starts with the number $\alpha=d v^{\nu}-1$ and the class $\mathcal{R}$ of subsets $S$ of $\Lambda$ such that $\bar{\mu}(S) \leq \alpha$.

Branch and Bound Procedure (BB1) (according to $\Lambda_{1}$ )

$\langle\langle$ Initialization $\rangle\rangle$

Set $k \leftarrow 0$ and $\mathcal{R}_{0} \leftarrow \mathcal{R}$.

$\langle\langle$ Step $k\rangle\rangle$

$\langle k 1\rangle$ Set $\mu_{k} \leftarrow \min \left\{\bar{\mu}(S) \mid S \in \mathcal{R}_{k}\right\}$ and choose $S_{k} \in \mathcal{R}_{k}$ such that $\bar{\mu}\left(S_{k}\right)=\mu_{k}$. Divide $S_{k}$ into $S_{k 1}, \ldots, S_{k p}$ by an integral rectangular division and set $\mathcal{R}_{k} \leftarrow$ $\mathcal{R}_{k} \backslash\left\{S_{k}\right\} \cup\left\{S_{k 1}, \ldots, S_{k p}\right\}$,

$\langle k 2\rangle$ For $j=1, \ldots, p$ do:

(a) If $\left|S_{k j} \cap \mathbb{Z}_{n}\right|=1$, then do:

(i) Set $\mathcal{R}_{k} \leftarrow \mathcal{R}_{k} \backslash\left\{S_{k j}\right\}$.

(ii) Choose $\lambda \in S_{k j} \cap \mathbb{Z}_{n}$ and solve $\max \{\lambda x \mid x \in X\}$, yielding the optimal face $F(\lambda)$.

(iii) Solve $\min \{d x \mid x \in F(\lambda)\}$, yielding a vertex solution $x(\lambda)$. 
(iv) If $d x(\lambda) \leq \alpha$, then set $w \leftarrow x(\lambda)$ and go to $\langle k 4\rangle$. Otherwise, go to Endfor.

(b) If $\left|S_{k j} \cap \mathbb{Z}_{n}\right|>1$, then do:

(i) Solve $\bar{P}^{\prime}\left(S_{k j}\right)$, yielding the optimal value $\bar{\mu}\left(S_{k j}\right)$ and an optimal solution $y$ if feasible $\left(\bar{\mu}\left(S_{k j}\right)=+\infty\right.$ when infeasible).

(ii) If $\bar{\mu}\left(S_{k j}\right)>\alpha$, then set $\mathcal{R}_{k} \leftarrow \mathcal{R}_{k} \backslash\left\{S_{k j}\right\}$ and go to Endfor.

(iii) Identify the minimal face $F$ of $X$ containing $y$, solve $\min \{d x \mid x \in F\}$

Endfor for a vertex solution $w$ and go to $\langle k 4\rangle$.

$\langle k 3\rangle$ If $\mathcal{R}_{k} \neq \emptyset$, then set $\mathcal{R}_{k+1} \leftarrow \mathcal{R}_{k}, k \leftarrow k+1$ and go to $\langle\langle$ Step $k\rangle\rangle$. Otherwise, set $\mathcal{R} \leftarrow \emptyset$ and quit.

$\langle k 4\rangle$ Set $\mathcal{R} \leftarrow\left\{S \mid S \in \mathcal{R}_{k} ; \bar{\mu}(S) \leq d w-1\right\}$ and quit.

Theorem 4.1. Procedure BB1 terminates after finitely many iterations, either yielding an extreme maximal flow with an objective function value being less than or equal to $\alpha$, or indicating that such a maximal flow does not exist. In other words, Procedure BB1 solves Problem $C G O(\alpha)$ finitely.

Proof. Each subsequence of rectangles, $\left\{S_{q}\right\}$, generated throughout Procedure (BB1) such that $S_{q+1} \subset S_{q} \forall q$, must be finite, since every $S_{q}$ contains at least one element from $\mathbb{Z}_{n}$.

If the procedure terminates at Step $\langle k 4\rangle$, then the point $w$ is an extreme maximal flow satisfying $d w \leq \alpha$.

If the procedure terminates at Step $\langle k 3\rangle$, i.e., $\mathcal{R}=\emptyset$, then it follows that each subset $S$ of $\Lambda_{1}$ yields a lower bound $\bar{\mu}(S)>\alpha$, which implies that there does not exist an extreme maximal flow $v \in X_{M} \cap X_{V}$ such that $d v \leq \alpha$.

Branch and Bound Procedure (BB2) (according to $\Lambda_{2}$ )

$\langle\langle$ Initialization $\rangle\rangle$

Set $k \leftarrow 0$ and $\mathcal{R}_{0} \leftarrow \mathcal{R}$.

$\langle\langle$ Step $k\rangle\rangle$

$\langle k 1\rangle$ (a) Set $\mu_{k} \leftarrow \min \left\{\bar{\mu}(S) \mid S \in \mathcal{R}_{k}\right\}$ and choose $S_{k} \in \mathcal{R}_{k}$ such that $\bar{\mu}\left(S_{k}\right)=\mu_{k}$.

(b) Divide $S_{k}$ into $S_{k 1}, \ldots, S_{k p}$ by a simplicial division and set

$\mathcal{R}_{k} \leftarrow \mathcal{R}_{k} \backslash\left\{S_{k}\right\} \cup\left\{S_{k 1}, \ldots, S_{k p}\right\}$,

$\langle k 2\rangle$ For $j=1, \ldots, p$ do:

(a) Solve $\bar{P}\left(S_{k j}\right)$, yielding the optimal value $\bar{\mu}\left(S_{k j}\right)$ and an optimal solution $\left(x_{k j}^{1}, \ldots, x_{k j}^{n}\right)$ if feasible $\left(\bar{\mu}\left(S_{k j}\right)=+\infty\right.$ when infeasible $)$.

(b) If $\bar{\mu}\left(S_{k j}\right)>\alpha$, then set $\mathcal{R}_{k} \leftarrow \mathcal{R}_{k} \backslash\left\{S_{k j}\right\}$.

(c) Let $x_{k j} \leftarrow \sum_{\ell=1}^{n} x_{k j}^{\ell}$.

(d) If $x_{k j} \in X_{M}$, then set $y \leftarrow x_{k j}$ and go to $\langle k 4\rangle$.

Endfor

$\langle k 3\rangle$ (a) If $\mathcal{R}_{k} \neq \emptyset$, then set $\mathcal{R}_{k+1} \leftarrow \mathcal{R}_{k}, k \leftarrow k+1$ and go to $\langle k 1\rangle$.

(b) Otherwise, set $\mathcal{R} \leftarrow \emptyset$ and quit.

$\langle k 4\rangle$ (a) Identify the minimal face $F$ of $X$ containing $y$ and solve

$$
\min \{d x \mid x \in F\}
$$


for a vertex solution $w$.

(b) Set

$$
\mathcal{R} \leftarrow\left\{S \mid S \in \mathcal{R}_{k} ; \bar{\mu}(S) \leq d w-1\right\},
$$

and quit.

If the Procedure BB2 terminates after finitely many iterations, then it either yields an extreme maximal flow with an objective function value being less than or equal to $\alpha$, or indicates that such a maximal flow does not exist when $\mathcal{R}=\emptyset$.

In the case that the procedure is infinite, it generates at least an infinite nested subsequence $\left\{S_{\kappa}\right\}$ of subsimplices such that $S_{\kappa+1} \subset S_{\kappa}$ for all $\kappa$. Below we show that the procedure will generate an infinite sequence of flows converging to a minimum maximal flow, i.e., an optimal solution of Problem $P$.

We recall the exhaustiveness of the division process introduced for the establishment of convergence properties of branch and bound algorithms in global optimization (see, e.g., Thoai and Tuy [31]). An infinite nested sequence of subsets $\left\{S_{\kappa}\right\}_{\kappa}$ is said to be exhaustive if $\bigcap_{\kappa=1}^{\infty} S_{\kappa}$ is a singleton. A simplicial division process is called exhaustive if each nested infinite subsequence of subsets generated by it is exhaustive. It is well known that the simplicial bisection process is exhaustive.

Theorem 4.2. Assume that the division process is exhaustive and Procedure BB2 is infinite. For each $k$ let $x_{k}=\sum_{\ell=1}^{n} x_{k}^{\ell}$, where $\left(x_{k}^{1}, \ldots, x_{k}^{n}\right)$ is an optimal solution to $\bar{P}\left(S_{k}\right)$. Then the sequence $\left\{x_{k}\right\}$ has an accumulation point, and each of them is an optimal solution of Problem $P$.

Proof. Since $x_{k} \in X$ for each $k$ and $X$ is a compact set, the sequence $\left\{x_{k}\right\}$ has an accumulation point in $X$. Let $x^{*}$ be an arbitrary accumulation point of $\left\{x_{k}\right\}$, and let $\left\{x_{\kappa}\right\}$ be a subsequence converging to $x^{*}$. Fom Lemma 3.5 and the property that $\left\{\mu_{\kappa}\right\}$ is nondecreasing and bounded from above by $\alpha$, it follows that there exists a limit $\mu^{*}$ of $\left\{\mu_{\kappa}\right\}$. By using subsequences if necessary, assume that $\mu_{\kappa} \rightarrow \mu^{*}$ as $\kappa \rightarrow \infty$, and $\left\{S_{\kappa}\right\}$ is the corresponding subsequence of simplices such that $S_{\kappa+1} \subset S_{\kappa}$ for all $\kappa$. Since the simplicial division is exhaustive, it follows that

$$
\bigcap_{\kappa=1}^{\infty} S_{\kappa}=\left\{\lambda^{*}\right\}
$$

and hence, denoting by $\lambda_{\kappa}^{\ell}(\ell=1, \ldots, n)$ the vertices of $S_{\kappa}$ we have $\lambda_{\kappa}^{\ell} \rightarrow \lambda^{*} \in \Lambda_{2}$ as $\kappa \rightarrow \infty$ for $i=1, \cdots, n$. Thus, we have $x^{*} \in X, \lambda^{*} \in \Lambda_{2}$, and $\lambda^{*} x^{*}-\phi\left(\lambda^{*}\right) \geq 0$, which implies that $\left(x^{*}, \lambda^{*}\right)$ is a feasible solution of Problem $P\left(\Lambda_{2}\right)$, i.e. Problem $P$, and therefore, an optimal solution of this problem with the optimal value $d x^{*}=\mu^{*}$.

\section{Global Optimization Algorithm and Approximate Optimal Solution}

Combining Procedure AVS with Procedure BB1 or BB2 in Section 4, we propose the following algorithm for globally solving Problem $P$.

\section{Global Optimization Algorithm (GOA)}

$\langle\langle$ Initialization $\rangle\rangle$ 
Compute an extreme maximal flow $w^{0} \in X_{V} \cap X_{M}$. If $N_{M}\left(w^{0}\right)=\emptyset$, then $w^{0}$ is an optimal solution of $P$. Otherwise, set $\nu \leftarrow 1, \mathcal{R} \leftarrow\{\Lambda\}$ and go to Iteration $\nu$.

$\langle\langle$ Iteration $\nu\rangle\rangle$

$\langle\nu 1\rangle$ Apply AVS to Problem $P$ starting from $w^{\nu-1}$ and let $v^{\nu}$ be the extreme maximal flow obtained. Set $\alpha_{\nu} \leftarrow d v^{\nu}-1$ and go to Step $\nu 2$.

$\langle\nu 2\rangle$ Set $\mathcal{R} \leftarrow\left\{S \mid S \in \mathcal{R} ; \bar{\mu}(S) \leq \alpha_{\nu}\right\}$ and apply BB.

(a) If an extreme maximal flow $w^{\nu}$ with $d w^{\nu} \leq \alpha_{\nu}$ is found, set $\nu \leftarrow \nu+1$ and go to Iteration $\nu$.

(b) If $\mathrm{BB}$ terminates with an empty $\mathcal{R}$, then stop (the point $v^{\nu}$ is an optimal solution of $P$ ).

When BB2 is used in the algoithm GOA, it can be infinite. For the case that Procedure BB2 is infinite, we introduce the following concept of approximate optimal solutions of Problem $P$.

Definition 5.1. Given a real numbers $\gamma>0$, a flow $\bar{x}$ is called a $\gamma$-optimal solution of Problem $P$ if it satisfies the following conditions:

(i) There exists $\bar{\lambda} \in \Lambda$ such that $\bar{\lambda} \bar{x}-\phi(\bar{\lambda}) \geq-\gamma$, and

(ii) $d \bar{x}$ is a lower bound of the optimal value of Problem $P$.

Using this concept, we modify Procedure BB2 slightly to obtain the finiteness of the global optimiztion algorithm. Recall that $\lambda_{k}^{1}, \ldots, \lambda_{k}^{n}$ are the vertices of $S_{k}$ and $\left(x_{k}^{1}, \cdots, x_{k}^{n}\right)$ is an optimal solution of Problem $\bar{P}\left(S_{k}\right)$. The modification consists of the following additional stopping criterion between (a) and (b) at step $\langle k 1\rangle$.

$$
\text { If } \sum_{\ell=1}^{n} \lambda_{k}^{\ell} x_{k}^{\ell}-\Phi\left(S_{k}\right) \geq-\delta, \text { then stop. }
$$

We will show in Theorem 5.2 below that $x_{k}=\sum_{\ell=1}^{n} x_{k}^{\ell}$ is an approximate optimal solution of Problem $P$ in the sense of Definition 5.1.

Theorem 5.2. Assume that within Procedure BB2 the simplicial division is exhaustive, and the additional stopping criterion (5.1) is used at Step $\langle k 1\rangle$. Then

(i) The global optimization algorithm always terminates after finitely many iterations; and

(ii) If Procedure BB2 terminates by the additional stopping criterion (5.1), then $x_{k}=\sum_{\ell=1}^{n} x_{k}^{\ell}$ is a $(\delta+n \varepsilon)$-optimal solution of Problem P, where

$$
\varepsilon=\max \left\{\left(\lambda_{k}^{\ell}-\lambda_{k}^{\ell^{\prime}}\right) x_{k}^{\ell} \mid \ell, \ell^{\prime}=1, \ldots, n\right\} .
$$

Proof. We show that Procedure BB2 is finite. Then the finiteness of GOA follows immediately.

Suppose Procedure BB2 could be infinite. Then from Theorem 4.2, it would generate an infinite sequence $\left\{x_{\kappa}\right\}$ converging to an optimal solution of Problem $P$. This implies that there exists an index $\bar{\kappa}$ such that

$$
\sum_{\ell=1}^{n} \lambda_{\bar{\kappa}}^{\ell} x_{\bar{\kappa}}^{\ell}-\Phi\left(S_{\bar{\kappa}}\right) \geq-\delta
$$


Thus, Procedure BB2 must stop at iteration $\bar{\kappa}$.

Assume now Procedure BB2 terminates at step $\langle k 1\rangle$ of Iteration $k$ by stopping criterion (5.1). From (5.2) it follows that for each $\ell=1, \ldots, n$ and for any $\lambda \in S_{k}$ we have

$$
\lambda_{k}^{\ell} x_{k}^{\ell}-\lambda x_{k}^{\ell} \leq \varepsilon
$$

which implies

$$
\lambda \sum_{\ell=1}^{n} x_{k}^{\ell} \geq \sum_{\ell=1}^{n} \lambda_{k}^{\ell} x_{k}^{\ell}-n \varepsilon .
$$

Let now $\bar{\lambda} \in S_{k}$ such that $\phi(\bar{\lambda})=\Phi\left(S_{k}\right)$. Then

$$
\bar{\lambda} x_{k}-\phi(\bar{\lambda})=\bar{\lambda} \sum_{\ell=1}^{n} x_{k}^{\ell}-\phi(\bar{\lambda}) \geq \sum_{\ell=1}^{n} \lambda_{k}^{\ell} x_{k}^{\ell}-\Phi\left(S_{k}\right)-n \varepsilon,
$$

which implies by (5.1) that

$$
\bar{\lambda} x_{k}-\phi(\bar{\lambda}) \geq-(\delta+n \varepsilon) .
$$

Note that $d x_{k}=\bar{\mu}\left(S_{k}\right)$ is a lower bound of the optimal value of $P$ by the choice of $S_{k}$. Then by Definition $5.1 x_{k}$ is a $(\delta+n \varepsilon)$-optimal solution of Problem $P$.

\section{Conclusions}

In this article we propose an algorithm to combine different techniques in local search anad global optimization for solving the minimum maximal flow problem. The characteristic property of this algorithm is that the advantageous network flow structure is fully exploited. A detailed implementation and comparison of different procudures will be presented in a subsequent paper.

\section{REFERENCES}

[1] P.K. Ahuja, T.L. Magnanti and J.B. Orlin, Network Flows, Theory, Algorithms, and Applications, Prentice Hall, Englewood Cliffs, 1993.

[2] H.A. Lethi, D.T. Pham and L.D. Muu, "Numerical solution for optimization over the efficient set by d.c. optimization algorithms," Operations Research Letters 19 (1996) 117-128.

[3] K. Belling-Seib, P. Mevet, and C. Muller, "Network flow problems with one side constraint: a comparision of three solution methods," Computers and Operations Research 15 (1988) 381394.

[4] H.P. Benson, "An all-linear programming relaxation algorithm for optimizing over the efficient set," Journal of Global Optimization 1 (1991) 83-104.

[5] H.P. Benson, "A finite nonadjacent extreme-point search algorithm for optimization over the efficient set," Journal of Optimization Theory and Applications 73 (1992) 47-64.

[6] H.P. Benson, "A geometric analysis of the efficient outcome set in multiple objective convex program with linear criteria functions," Journal of Global Optimization 6 (1995) 213-251.

[7] H.P. Benson and D. Lee, "Outcome-based algorithm for optimizing over the efficient set of a bicriteria linear programming," Journal of Optimization Theory and Applications 88 (1996) $77-105$.

[8] H.P. Benson and S. Sayin, "Optimization over the efficient set: four special case," Journal of Optimization Theory and Applications 80 (1994) 3-18.

[9] S. Bolintineanu, "Minimization of a quasi-concave function over an efficient set," Mathematical Programming 61 (1993) 89-110. 
[10] N. Bryson, "Applications of the parametric programming procedure," European Journal of Operational Research 54 (1991) 66-73.

[11] J.P. Dauer and T.A. Fosnaugh, "Optimization over the efficient set," Journal of Global Optimization 7 (1995) 261-277.

[12] J.G. Ecker and J.H. Song, "Optimizing a linear function over an efficient set," Journal of Optimization Theory and Applications 83 (1994) 541-563.

[13] J. Fülöp, "A cutting plane algorithm for linear optimization over the efficient set," in: S.Komlösi, T.Rapcsàk and S.Shaible eds., Generalized Convexity, Lecture Notes in Economics and Mathematical Systems 405, (Springer-Verlag, Berlin, 1994) pp.374-385.

[14] M.R. Garay and D.S. Johnson, Computers and Intractability: A Guide to the Theory of NPCompleteness, (Freeman, San Francisco, 1979).

[15] R. Horst, P. Pardalos, and N.V. Thoai '-it Introduction to Global Optimization, 2nd Extended Edition, Kluwer Academic Publishers, Dordrecht, 2000.

[16] R. Horst and N.V. Thoai, "Maximizing a concave function over the efficient or weakly-efficient set," European Journal of Operational Research 117 (1999) 239-252.

[17] R. Horst and H. Tuy, Global Optimization: Deterministic Approach, (Springer, Berlin, 1996).

[18] M. Iri, "An essay in the theory of uncontrollable flows and congestion", Technical Report, Department of Information and System Engineering, Faculty of Science and Engineering, Chuo University, TRISE 94-03 (1994).

[19] M. Iri, "Network flow -theory and applications with practical impact," J.Doležal and J.Fidler eds., System Modelling and Optimization, (Chapman \& Hall, London, 1996) pp.24-36.

[20] L.D. Muu, "A convex-concave programming method for optimizing over the efficient set," Acta Mathematica Vietnamica 25 1, 67-85.

[21] P.H. Naccache, "Connectedness of the set of nondominated outcomes in multicriteria optimization," Journal of Optimization Theory and Applications 25 (1978) 459-467.

[22] J. Philip, "Algorithms for the vector maximization problem," Mathematical Programming 2 (1972) 207-229.

[23] T.Q. Phong and J.Q. Tuyen, "Bisection search algorithm for optimizing over the efficient set," to appear in Vietman Journal of Mathematics.

[24] R.T. Rockafellar and R.J-B. Wets, Variational Analysis. (Springer-Verlag, Berlin, 1998).

[25] S. Sayin, "Optimizing over the efficient set using a top-down search of faces," Operations Research 48 (2000) 65-72.

[26] Y. Sawaragi, H. Nakayama and T. Tanino, Theory of Multiobjective Optimization (Academic Press, Orland, 1985).

[27] J.M. Shi and Y. Yamamoto, "A global optimization method for minimum maximal flow problem," Acta Mathematica Vietnamica 22 (1997) 271-287.

[28] M. Shigeno, I. Takahashi and Y. Yamamoto, "Minimum Maximal Flow Problem - An Optimization over the Efficient Set -," Discussion Paper No.912, Institute of Policy and Planning Sciences, University of Tsukuba (March 2001).

[29] R.E. Steuer, Multiple Criteria Optimization: Theory, Computation and Application (Wiley, New York, 1985).

[30] P.T. Thach, H. Konno and D. Yokota, "Dual approach to nminimization on the set of paretooptimal solutions," Journal of Optimization Theory and Applications 88 (1996) 689-707.

[31] N.V. Thoai and H. Tuy "Convergent Algorithms for Minimizing a Concave Function", Mathematics of Operations Research 5 (1980) 556-566.

[32] N.V. Thoai, "A class of optimization problems over the efficient set of a multiple criteria nonlinear programming problem," European Journal of Operational Research 122 (2000) 5868.

[33] N.V. Thoai, "Conical algorithm in global optimization for optimizing over efficient sets," Journal of Global Optimization 18 (2000) 321-336.

[34] D.J. White, Optimality and Efficiency, (John Wiley \& Sons, Chichester, 1982).

[35] D.J. White, "The maximization of a function over the efficient set via a penalty function approach," European Journal of Operational Research 94 (1996) 143-153. 
[36] S. Yamada, T. Tanino and M. Inuiguchi, "An inner approximation method for optimization over the weakly efficient set," Journal of Global Optimization 16 (2000) 197-217.

[37] Y. Yamamoto, "Optimization over the efficient set: Overview," to appear in Journal of Global Optimization .

Institute of Policy and Planning Sciences, Univeristy of Tsukuba, Tsukuba, Ibaraki 305-8573, JAPAN

E-mail address: jgoto@sk.tsukuba.ac.jp, thoai@sk.tsukuba.ac.jp, thoai@uni-trier.de, yamamoto@sk.tsukuba.ac.jp 